\title{
Inhibitory Inputs from Rostromedial Tegmental Neurons Regulate Spontaneous Activity of Midbrain Dopamine Cells and Their Responses to Drugs of Abuse
}

\author{
Salvatore Lecca', Miriam Melis', Antonio Luchicchi', Anna Lisa Muntoni ${ }^{2,3}$ and Marco Pistis*,1,2,3 \\ 'BB Brodie Department of Neuroscience, University of Cagliari, Monserrato, Italy; ${ }^{2}$ Center of Excellence for the Neurobiology of Addiction, \\ University of Cagliari, Monserrato, Italy; ${ }^{3}$ CNR Neuroscience Institute-Cagliari, University of Cagliari, Monserrato, Italy
}

\begin{abstract}
The rostromedial tegmental nucleus (RMTg), a structure located just posterior to the ventral tegmental area (VTA), is an important site involved in aversion processes. The RMTg contains $\gamma$-aminobutyric acid neurons responding to noxious stimuli, densely innervated by the lateral habenula and providing a major inhibitory projection to reward-encoding dopamine (DA) neurons in the VTA. Here, we studied how RMTg neurons regulate both spontaneous firing of DA cells and their response to the cannabinoid agonist WIN552 I2-2 (WIN), morphine, cocaine, and nicotine. We utilized single-unit extracellular recordings in anesthetized rats and whole-cell patch clamp recordings in brain slices to study RMTg-induced inhibition of DA cells and inhibitory postsynaptic currents (IPSCs) evoked by stimulation of caudal afferents, respectively. The electrical stimulation of the RMTg elicited a complete suppression of spontaneous activity in approximately half of the DA neurons examined. RMTg-induced inhibition correlated with firing rate and pattern of DA neurons and with their response to a noxious stimulus, highlighting that inhibitory inputs from the RMTg strongly control spontaneous activity of DA cells. Both morphine and WIN depressed RMTg-induced inhibition of DA neurons in vivo and IPSCs evoked by RMTg stimulation in brain slices with presynaptic mechanisms. Conversely, neither cocaine nor nicotine modulated DA neuron responses to RMTg stimulation. Our results further support the role of the RMTg as one of the main inhibitory afferents to DA cells and suggest that cannabinoids and opioids might disinhibit DA neurons by profoundly influencing synaptic responses evoked by RMTg activation.

Neuropsychopharmacology (2012) 37, II64-I I76; doi:I0.1038/npp.20 II.302; published online I4 December 20 I I
\end{abstract}

Keywords: electrophysiology; patch clamp; cannabinoids; morphine; rat

\section{INTRODUCTION}

The mesopontine rostromedial tegmental nucleus (RMTg), also named tail of the ventral tegmental area (VTA; Kaufling et al, 2009; Perrotti et al, 2005), is a recently identified nucleus, primarily containing $\gamma$-aminobutyric acid (GABA) neurons projecting to midbrain dopamine (DA) neurons in the substantia nigra pars compacta and in the VTA (Jhou et al, 2009a; Kaufling et al, 2010a).

The RMTg has a pivotal role in processing both aversive and appetitive stimuli (Jhou et al, 2009a). In fact, RMTg neurons are excited by noxious stimuli and inhibited by rewarding stimuli (Hong et al, 2011; Jhou et al, 2009a) in a similar way to glutamatergic neurons of the lateral habenula

* Correspondence: Professor M Pistis, BB Brodie Department of Neuroscience, Center of Excellence for the Neurobiology of Addiction, University of Cagliari, Cittadella Universitaria, Monserrato, CA 09042, Italy, Tel: + 39070675 4324, Fax: + 39070675 4320, E-mail:mpistis@unica.it

Received 7 October 201 I; accepted 31 October 201 I
(LHb; Matsumoto and Hikosaka, 2007, 2009). This epithalamic area is involved in the mechanisms of fear, anxiety, and stress, and is also the main excitatory input to the RMTg (Jhou et al, 2009a). The LHb is one of the neural substrates for negative reinforcement and a source of inhibitory inputs to DA neurons (Christoph et al, 1986; Ji and Shepard, 2007; Matsumoto and Hikosaka, 2007). However, it was shown that the inhibitory influence of the LHb on midbrain DA cells requires an indirect mediation via RMTg neurons. These cells form inhibitory synapses with VTA DA neurons (Balcita-Pedicino et al, 2011) preferentially targeting the nucleus accumbens (Kaufling et al, 2010a). Consistently, electrical stimulation of the RMTg inhibits DA neurons both in rat (Lecca et al, 2011) and monkey (Hong et al, 2011).

Taken together, these studies suggest a functional integration between these areas mediating aversion and gratification. Considering that the electrical activity of DA neurons, and the resulting DA output in terminal areas, depends on the balance between excitatory and inhibitory inputs (Marinelli et al, 2006; Morikawa and Paladini, 2011), it is of great interest to characterize the impact of this 
pathway on the activity of DA neurons and on their responses to addicting drugs. In fact, recent studies indicate that abused substances affect RMTg neurons in different ways. Hence, these cells show high immunoreactivity against $\mu$-opioid receptors (Jhou et al, 2009a); they express FosB/deltaFosB (Kaufling et al, 2010a; Kaufling et al, 2010b; Perrotti et al, 2005) or c-Fos (Geisler et al, 2008; Jhou et al, 2009a) following both acute and chronic injection of psychostimulants, and their electrical activity is inhibited by acute administration of most of the drugs of abuse (Lecca et al, 2011).

Here, we investigate how addictive substances affect the evoked inhibitory responses of VTA DA neurons to RMTg activation. We took advantage of extracellular single-unit recordings in anesthetized rats together with whole-cell patch clamp recordings in brain slices. We first characterized the electrophysiological responses of DA cells to RMTg electrical stimulation. Furthermore, we studied the effects of several prototypical drugs of abuse, the type 1 cannabinoid (CB1) receptor agonist WIN55212-2 (WIN), the opioidreceptor agonist morphine, nicotine, and cocaine, on inhibition elicited by RMTg stimulation.

\section{MATERIALS AND METHODS}

\section{In vivo Experiments}

Subjects and surgery. Experiments were performed in strict accordance with the EEC Council Directive of 24 November 1986 (86/609). Male Sprague-Dawley albino rats (HarlanNossan, San Pietro al Natisone, Italy) weighing 250-350 g were used in all in vivo experiments. All efforts were made to minimize pain and suffering and to reduce the number of animals used. Animals were housed in groups of three to six in standard conditions of temperature and humidity under a $12 \mathrm{~h}$ light/dark cycle (with lights on at 0700 hours) with food and water available ad libitum. We anesthetized rats with urethane $(1300 \mathrm{mg} / \mathrm{kg}$, ip), cannulated their femoral vein for intravenous administration of pharmacological agents, and placed them in the stereotaxic apparatus (Kopf, Tujunga, CA), with their body temperature maintained at $37 \pm 1{ }^{\circ} \mathrm{C}$ by a heating pad. The scalp was retracted and one burr hole was drilled above the VTA $(5.6-5.8 \mathrm{~mm}$ posterior to bregma, $0.4-0.6 \mathrm{~mm}$ lateral to midline) for the placement of a recording electrode. To evaluate the inhibitory input arising from the RMTg to the VTA, a formvar-coated stimulating stainless steel bipolar electrode $(250 \mu \mathrm{m}$ tip diameter $)$ was aimed at the ipsilateral $\mathrm{RMTg}(7.2 \mathrm{~mm}$ posterior to bregma and $0.8 \mathrm{~mm}$ lateral to midline, and $6.5 \mathrm{~mm}$ ventral to cortical surface) according to the stereotaxic atlas of Paxinos and Watson (2007). The electrode was inserted with an inclination of $20^{\circ}$ anteroposterior on the coronal plane.

Single-unit recordings. Single unit activity of neurons located in the VTA (V 7.0-8.0 mm to cortical surface) was recorded extracellularly by glass micropipettes filled with $2 \%$ pontamine sky blue dissolved in $0.5 \mathrm{M}$ sodium acetate (impedance 2-5 M $\Omega$ ). Signal was filtered (band-pass 500$5000 \mathrm{~Hz}$ ) and amplified (Neurolog System, Digitimer, Hertfordshire, UK), displayed on a digital storage oscilloscope (TDS 3012, Tektronix, Marlow, UK), and digitally recorded. Experiments were sampled on- and off-line by a computer connected to CED Power 1401 laboratory interface (Cambridge Electronic Design, Cambridge, UK) running the Spike2 software (Cambridge Electronic Design). Single units were isolated and identified according to previously described electrophysiological characteristics (Grace and Bunney, 1983, 1984; Ungless et al, 2004). We recorded VTA DA neurons only when criteria for identification were fulfilled (firing rate $\leqslant 10 \mathrm{~Hz}$, duration of action potential $\geqslant 2.5 \mathrm{~ms}$ ).

Drugs were administered in bolus iv $(1 \mathrm{ml} / \mathrm{kg}$ of body weight). When drugs were administered, only one cell was recorded per rat. At the end of recording sessions, DC current ( $15 \mathrm{~mA}$ for $5 \mathrm{~min}$ ) was passed through the recording micropipette to eject pontamine sky blue for marking the recording site. Brains were then rapidly removed and fixed in $4 \%$ paraformaldehyde solution. The position of the electrodes was microscopically identified on serial sections $(60 \mu \mathrm{m})$ stained with neutral red.

Stimulation protocol. The experimental protocol was essentially as described previously (Lecca et al, 2011). Briefly, once a cell was selected, electrical stimuli consisting of single, monophasic, rectangular pulses $(0.5 \mathrm{~mA}, 0.5 \mathrm{~ms})$ were delivered to the RMTg at $1 \mathrm{~Hz}$. Responses to electrical stimulation of the RMTg were evaluated, and a peristimulus time histogram (PSTH) was generated online for each neuron.

Data analysis. Isolated DA neurons were recorded for $2 \mathrm{~min}$ to establish basal firing properties. Spontaneous firing rate, percentage of spikes in bursts, and coefficient of variation $(\mathrm{CV}=$ standard deviation of interspike intervals divided by the mean interspike interval; a measure of firing regularity) were determined. Additionally, autocorrelograms were generated using a $10 \mathrm{~ms}$ bin width for intervals up to $2 \mathrm{~s}$, to qualitatively classify neurons as firing in the regular, irregular or burst firing mode (Fabricius et al, 2010; Hyland et al, 2002). Autocorrelograms showing three or more regularly occurring peaks were characteristic of the regular firing pattern. An initial trough that rose smoothly to a steady state was classified as irregular firing pattern, whereas an initial peak, followed by decay to a steady state, was characteristic of the bursting mode (Fabricius et al, 2010; Hyland et al, 2002). After recording baseline activity, each cell was tested for its response to repetitive $(1 \mathrm{~Hz})$ stimulation of the RMTg. PSTHs were built from 100 stimuli and displayed using $1 \mathrm{~ms}$ bin width. A cell was considered inhibited or excited when the number of action potentials/ bin (bin length $=1 \mathrm{~ms}$ ) in the $50 \mathrm{~ms}$ after the stimulus was significantly lower or higher (one-way ANOVA for repeated measures), respectively, than baseline levels (the number of action potentials/bin in the $50 \mathrm{~ms}$ period before the stimulus). The duration of stimulus-evoked inhibition was defined as the time of complete cessation of firing after the stimulus.

\section{In vitro Experiments}

The preparation of VTA slices was as described previously (Melis et al, 2002). Briefly, Sprague-Dawley rats (10-25 days, Harlan-Nossan) were anesthetized with halothane and killed. A block of tissue containing the midbrain was sliced 
in the horizontal plane $(300 \mu \mathrm{m})$ with a vibratome (Leica, Nussloch, Germany) in ice-cold low $\mathrm{Ca}^{2+}$ solution containing (in mM): $126 \mathrm{NaCl}, 1.6 \mathrm{KCl}, 1.2 \mathrm{NaH}_{2} \mathrm{PO}_{4}, 1.2 \mathrm{MgCl}_{2}$, $0.625 \mathrm{CaCl}_{2}, 18 \mathrm{NaHCO}_{3}$, and 11 glucose. Slices (two per animal) were transferred in a holding chamber and allowed to recover for at least $1 \mathrm{~h}$ before being placed in the recording chamber and superfused with a bicarbonate-buffered solution $\left(32-34{ }^{\circ} \mathrm{C}\right)$ saturated with $95 \% \mathrm{O}_{2} / 5 \% \mathrm{CO}_{2}$ and containing (in mM): $126 \mathrm{NaCl}, 1.6 \mathrm{KCl}, 1.2 \mathrm{NaH}_{2} \mathrm{PO}_{4}, 1.2$ $\mathrm{MgCl}_{2}, 2.4 \mathrm{CaCl}_{2}, 18 \mathrm{NaHCO}_{3}$, and 11 glucose.

Whole-cell recording. Cells were visualized with an upright microscope with infrared illumination, and whole-cell voltage clamp recordings were made by using an Axopatch 200B amplifier (Molecular Devices, CA). All GABA inhibitory postsynaptic currents (IPSCs) recordings were made with electrodes filled with an internal solution containing the following (mM): $\mathrm{KCl} 144,1 \mathrm{CaCl}_{2}, 3.45$ $\mathrm{K}_{4}$ BAPTA, 10 HEPES, $2 \mathrm{Mg}_{2}$ ATP, $0.25 \mathrm{Mg}_{2} \mathrm{GTP}, \mathrm{pH}$ 7.2-7.4. Experiments were begun only after series resistance had stabilized (typically 15-40 M $\Omega$ ). Series resistance and input resistance were monitored continuously online with a $4-\mathrm{mV}$ depolarizing step $(25 \mathrm{~ms})$. Data were filtered at $2 \mathrm{kHz}$, digitized at $10 \mathrm{kHz}$, and collected online with acquisition software (Clampex 8.2, Molecular Devices). Because of the composition of the internal solution, the $\mathrm{GABA}_{\mathrm{A}}$ IPSCs were inward at a membrane potential of $-70 \mathrm{mV}$ and were completely blocked by picrotoxin ( $100 \mu \mathrm{M}$, data not shown). DA cells were identified by the presence of a large $I_{\mathrm{h}}$ current (Johnson and North, 1992) that was assayed immediately after break-in, using a series of incremental $10 \mathrm{mV}$ hyperpolarizing steps from a holding potential of $-70 \mathrm{mV}$. A bipolar stainless steel stimulating electrode was placed 450-650 $\mu \mathrm{m}$ caudal to the recording electrode and was used to stimulate at a frequency of $0.1 \mathrm{~Hz}(50 \mu \mathrm{s}$ duration). Neurons were voltage clamped at a membrane potential of $-70 \mathrm{mV}$. All $\mathrm{GABA}_{\mathrm{A}}$ IPSCs were recorded in presence of 2-amino-5-phosphonopentanoic acid $(100 \mu \mathrm{M})$, 6-cyano2,3-dihydroxy-7-nitroquinoxaline $(10 \mu \mathrm{M})$, strychnine $(1 \mu \mathrm{M})$, and eticlopride $(100 \mathrm{nM})$ to block $N$-methyl-D-aspartate, $\alpha$-amino-3-hydroxy-5-methyl-isoxazolepropionic acid, glycine, and DA D2-mediated synaptic currents, respectively. As already described (Bonci and Williams, 1997), there was no effect of this solution on the holding current of the DA cells.

\section{Drugs}

Morphine and cocaine hydrochloride were purchased from Salars (Como, Italy) and Akzo Pharma Division Diosynth (Oss, Netherlands), respectively. Nicotine ((-)-nicotine hydrogen tartrate) was purchased from Sigma (Milano, Italy). WIN and AM281 were purchased from Tocris (Bristol, UK). Naloxone was purchased from RBI (Natick, MA). Rimonabant (SR141716) was a generous gift of SanofiAventis Recherche (Montpellier, France). For experiments in vivo, WIN and rimonabant were emulsified in $1 \%$ Tween 80 , then diluted in saline and sonicated. All other drugs were diluted in saline. Nicotine solution was adjusted to $\mathrm{pH}=7.0$ with $\mathrm{NaOH}$. For experiments in vitro, all drugs were applied in known concentrations to the superfusion medium and, with the exception of WIN, AM281, and rimonabant were dissolved in saline. WIN, rimonabant and AM281 were dissolved in DMSO as stock concentrations and the final concentration of DMSO was $<0.01 \%$.

\section{Statistical Analysis}

Changes in the duration of inhibition evoked by RMTg stimulation were calculated analyzing PSTH every $100 \mathrm{~s}$. We averaged the effects of the drugs for the $100 \mathrm{~s}$ period following drug administration and compared them with the mean of the predrug baseline. All the numerical data are given as mean \pm SEM. Data were compared and analyzed by using two-way ANOVA for repeated measures (treatment $\times$ time), or one-way ANOVA or Student's $t$ test for repeated measures. Correlation analysis was used when appropriate. Post hoc multiple comparisons were made using the Tukey's, Dunnett's, and Bonferroni's tests. Statistical analysis was performed by means of the Graphpad Prism software (La Jolla, CA).

The amplitudes of IPSCs were calculated by taking the $1 \mathrm{~ms}$ window around the peak of the IPSC and comparing this with the $2 \mathrm{~ms}$ window immediately before the stimulation artifact. Drugs were applied in known concentrations to the superfusion medium. Results in the text and Figures are presented as the mean \pm SEM. Results between groups were compared using two-way ANOVA. $P<0.05$ was considered as statistically significant.

\section{RESULTS}

\section{Effects of RMTg Stimulation on the Spontaneous Activity of VTA DA Neurons}

Given that RMTg GABA neurons diffusely project to midbrain DA cells (Jhou et al, 2009b), and that their electrical stimulation produces a temporary but complete inhibition of the spontaneous activity of VTA DA neurons (Lecca et al, 2011), we further characterized the inhibitory contribution from RMTg afferents to VTA DA neurons. To this aim, a sampling of DA neurons was carried out in six rats. We recorded the spontaneous activity of 60 cells in the posterior VTA identified as putative DA on the basis of their action potential characteristics (see Figure 1a for a representative example) and firing properties (see Figure 2a). Recording sites were located within the mediolateral aspects of the parabrachial pigmented nuclei of the VTA (Figure 1a). Although the VTA shows some cellular heterogeneity (Ford, 2006; Lammel et al, 2008; Margolis et al, 2008; Yamaguchi et al, 2011), the subregion where all recording sites were found contains a larger density of TH-positive neurons when compared with the more medial levels of the VTA (Yamaguchi et al, 2011). After $2 \mathrm{~min}$ of stable baseline firing rate, we tested the response of DA neurons to RMTg stimulation $(1 \mathrm{~Hz}, 0.5 \mathrm{~ms}$; Figure 1b). A cell was considered inhibited or excited when the number of action potentials/bin (bin length $=1 \mathrm{~ms}$ ) in the $50 \mathrm{~ms}$ after the stimulus was significantly lower or higher (one-way ANOVA for repeated measures), respectively, than that calculated in the $50 \mathrm{~ms}$ before the stimulus. The electrical stimulation of the RMTg induced an inhibition of the spontaneous activity in $53.3 \%$ of the neurons examined (32 out of 60; Figure 1b). A representative PSTH 
a
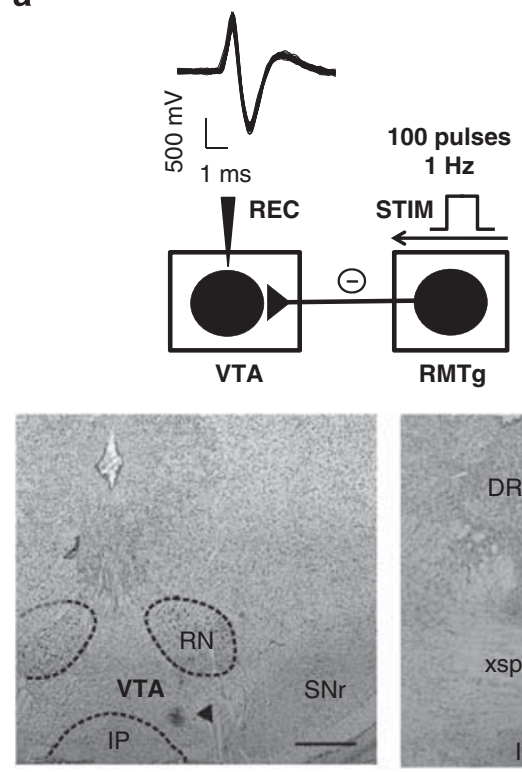

b

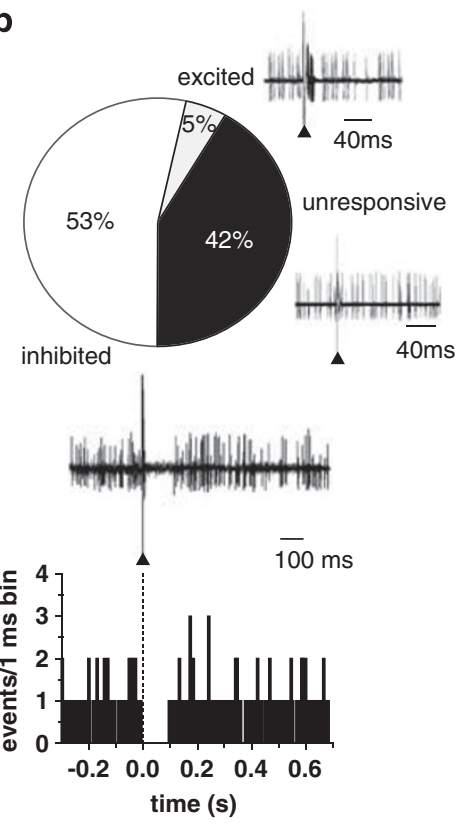

Figure I RMTg stimulation induces inhibition of VTA DA neuron spontaneous activity. (a; top panel) The trace shows typical superimposed spike waveforms of a DA neuron recorded in the VTA. Below, the diagram illustrates the stimulation protocol used in all in vivo experiments. The RMTg was stimulated with rectangular current pulses $(0.5 \mathrm{~mA}, 0.5 \mathrm{~ms})$ delivered at I Hz, and responses of DA cells to RMTg stimulation were recorded extracellularly. Recording location for a DA neuron in the posterior VTA (the triangle indicates the pontamine sky blue dye; bottom panel; left). Right: electrical stimulation site in the RMTg. The black asterisk indicates the area lesioned by the tip of the electrode, the black triangles indicate the track of the recording electrode. $\mathrm{DR}$, dorsal raphe; IP, interpeduncular nucleus; RN, red nucleus; SNr, substantia nigra pars reticulata; xspc, decussation of the superior cerebellar peduncle. Scale bars, $0.5 \mathrm{~mm}$. (b) Percentages of different types of DA cell responses to RMTg stimulation. Traces acquired from a digital oscilloscope represent typical examples of excited, inhibited, and unresponsive DA cells. A PSTH of the inhibited cell is displayed (I00 consecutive sweeps) and shows that RMTg stimulation produced a full suppression in firing activity of a VTA DA neuron for $\sim 90 \mathrm{~ms}$. Note that the inhibitory response occurred immediately after the stimulus (arrowhead).

illustrating the typical RMTg-induced suppression of VTA DA neuronal discharge is shown in Figure 1b. Owing to the close proximity between the stimulating and the recording sites, we were unable to calculate the onset latency of the inhibition. Hence, by assuming a conduction velocity of $\sim 2$ $\mathrm{m} / \mathrm{s}$, typical for fast-firing GABA neurons (Jones et al, 1999), the latency would be $<3 \mathrm{~ms}$, and would fall within the duration of the stimulus artifact. This short latency was consistent with a direct innervation, and persisted for $82.5 \pm 6.12 \mathrm{~ms}$ (range, $28-250 \mathrm{~ms} ; n=32$; Figure $1 \mathrm{~b}$ ). Among VTA DA cells that were not inhibited $(n=28), 25$ were unresponsive to RMTg stimulation (42\%), and 3 others were excited (5\%) (Figure 1b). To confirm that the placement of the stimulating electrode allowed a specific stimulation of RMTg neurons, in a separate set of experiments (in four rats) the electrode was placed $1 \mathrm{~mm}$ more laterally to avoid the RMTg (coordinates in $\mathrm{mm}$ : -7.2 posterior to bregma; 1.8 lateral to midline; and 6.5 ventral from cortical surface). Under these circumstances, the responses of VTA neurons were drastically different $\left(\chi^{2}=27.88 ; \mathrm{df}=2 ; P<0.0001\right.$, $\chi^{2}$-test), the effect of electrical stimulation being: $5.2 \%$ (1/19) of cells inhibited, $52.6 \%(10 / 19)$ excited, and $42.1 \%$ $(8 / 19)$ unresponsive.

Correlation between DA neuron spontaneous activity and duration of RMTg-evoked inhibition. In order to assess whether spontaneous firing activity of VTA DA neurons and the duration of inhibition evoked by RMTg stimulation were correlated, we analyzed a total of 69 DA cells inhibited from RMTg. In line with previous results in urethaneanesthetized rats (Kelland et al, 1990; Pistis et al, 2004), DA neurons fired at $3.7 \pm 0.2 \mathrm{~Hz} \quad(n=69)$, and presented $18.5 \pm 2.8 \%$ of spikes in bursts (data not shown). The mean $\mathrm{CV}$ of interspike intervals was $62.4 \pm 3.4 \%$, indicating that the average firing pattern of recorded VTA DA cells was irregular.

Interestingly, as illustrated in Figure $2 \mathrm{a}$, we found a negative correlation between duration of inhibition and both spontaneous firing rate $\left(\mathrm{F}_{1,67}=66.71 ; r=-0.71\right.$, $P<0.0001$, Pearson's test) and percentage of burst firing $\left(\mathrm{F}_{1,67}=4.05 ; r=-0.24, P<0.05\right.$, Pearson's test, data not shown), but not with $\mathrm{CV}\left(\mathrm{F}_{1,67}=1.83 ; r=0.16, P>0.05\right.$, Pearson's test, data not shown).

Moreover, generation of autocorrelograms for all recorded cells permitted their classification as regular, irregular, or bursting (Figure $2 \mathrm{~b}$ ). The distribution of these firing patterns confirmed that the majority of DA neurons (34 out of $69 ; 49.3 \%$ ) fired irregularly, whereas the remaining were classified as regular (14 out of $69 ; 20.3 \%$ ) or bursting neurons (21 out of $69 ; 30.4 \%$; see Figure $2 \mathrm{~b}$ ). Noteworthy, neurons showing an irregular discharge pattern exhibited a longer duration of inhibition (103.2 \pm $9.4 \mathrm{~ms} ; n=34)$ in response to RMTg stimulation than regular $(61.21 \pm 5.9 \mathrm{~ms} ; n=14)$ or bursting $(63.0 \pm 9.7 \mathrm{~ms}$; $n=21)$ DA cells $\left(\mathrm{F}_{2,66}=6.48, P<0.01\right.$, one-way ANOVA, and Tukey's test; Figure $2 \mathrm{~b}$ ).

Considering the role of RMTg in aversion, which is strongly supported by the predominant, phasic excitation of 

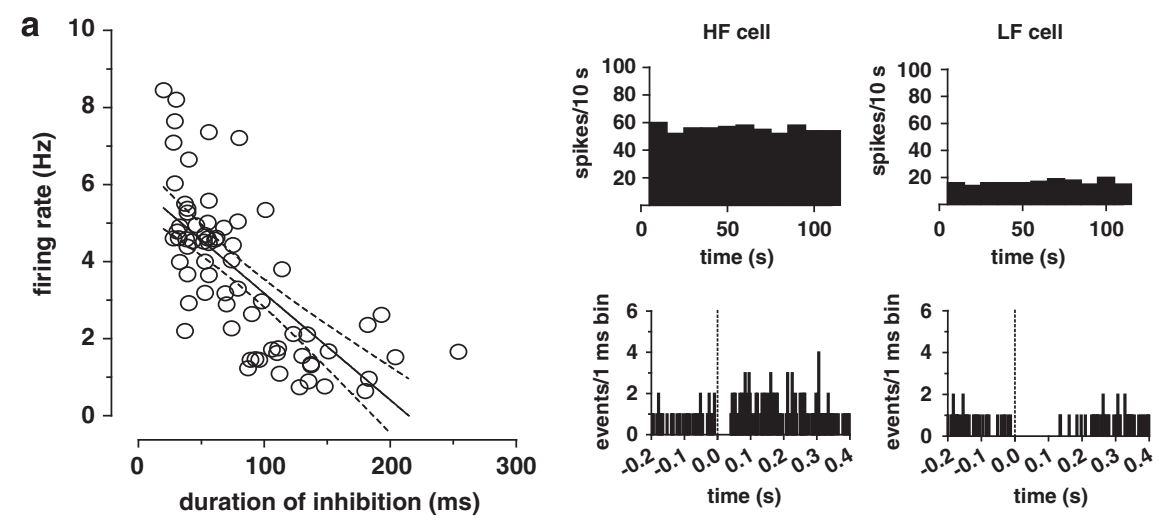

b
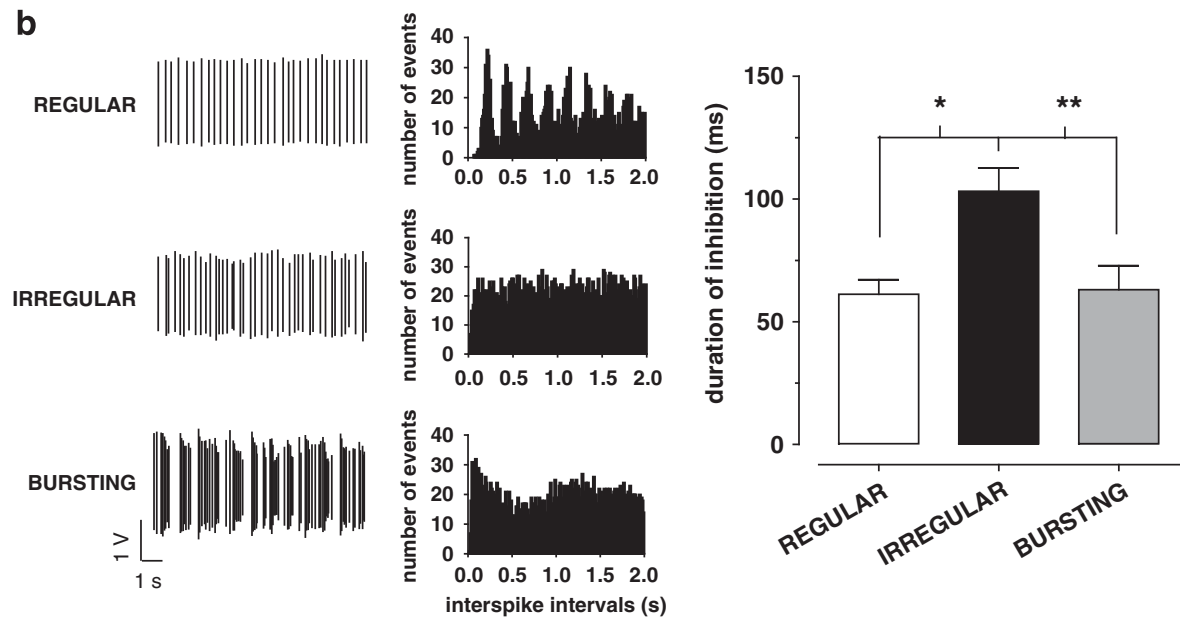

Figure 2 The duration of RMTg-evoked inhibition is negatively correlated with the spontaneous discharge activity of VTA DA neurons. (a) On the left, the graph illustrates the negative correlation between spontaneous firing rate and duration of inhibition induced by RMTg stimulation (dashed lines represent $\pm 95 \%$ confidence intervals). Representative rate histograms (top right) and PSTHs (bottom right) of two individual DA cells displaying that a high firing neuron (HF cell) was suppressed for a shorter period of time than a low firing neuron (LF cell). (b) Autocorrelograms of DA neurons were generated from action potential recordings and allowed the classification of their firing pattern into regular (top), irregular (middle), and bursting (bottom) mode. The right bar graph shows that irregular neurons exhibited a longer inhibitory response to RMTg stimulation when compared with regular or bursting cells. * $P<0.05$, **P $<0.0$ I, one-way ANOVA, and Tukey's test.

RMTg neurons in response to noxious stimuli (Jhou et al, 2009a; Lecca $e t$ al, 2011), and the heterogeneous responses of VTA DA neurons to aversive stimuli (Brischoux et al, 2009), we then examined whether this heterogeneity could depend on DA cell responses to RMTg stimulation. To this aim, the response to a brief $(2 \mathrm{~s})$ paw pinch (Lecca et al, 2011) was studied in 20 DA neurons (12 inhibited and 8 unresponsive). To detect rapid changes in firing rate, rate histograms were built with $0.5 \mathrm{~s}$ bins (Figure 3 ). The firing rate during the $5 \mathrm{~s}$ following the pinch (10 bins) was analyzed and compared with the average baseline values. Remarkably, neurons inhibited by RMTg stimulation responded to paw pinch with a pronounced short-lasting phasic inhibition (average firing rate after pinch: 78.4 \pm $5.6 \%$ of baseline, $n=12, \mathrm{~F}_{10,110}=2.06, P=0.03$, one-way ANOVA, and Dunnett's test). No significant change in firing rate was produced by paw pinch in unresponsive DA neurons (average firing rate after pinch: $85.2 \pm 5.2 \%$ of baseline firing rate, $n=8, \mathrm{~F}_{10,70}=0.85, P=0.58$, one-way ANOVA, and Dunnett's test). Figure 3 illustrates the average rate histograms of RMTg-inhibited and not-inhibited cells. The phasic decrease in firing rate occurred immediately after mechanical pinch in those DA cells suppressed by
RMTg stimulation (Figure 3a). On the other hand, neurons that did not respond to RMTg stimulation showed no significant decrease in firing rate after the aversive somatosensory stimulus (Figure $3 \mathrm{~b}$ ).

\section{Effects of Drugs of Abuse on RMTg-Induced Inhibition of VTA DA Cells}

Next, we tested the effects of different drugs of abuse (ie, the $\mathrm{CB} 1$ agonist WIN, morphine, cocaine, and nicotine) on VTA inhibition evoked by stimulation of the RMTg.

The CB1 agonist WIN reduces the inhibition of VTA DA neurons. Inhibition of RMTg GABA neurons by the CB1 agonist WIN (Lecca et al, 2011) might be one of the mechanisms contributing to cannabinoid-induced excitation of midbrain DA cells in vivo (French et al, 1997; Gessa et al, 1998). We therefore investigated the influence of WIN on RMTg-evoked suppression of DA neuron firing. To this aim, DA neurons in the VTA inhibited by RMTg stimulation were selected (see Figure 4a). Cells were recorded for $2 \mathrm{~min}$ and then the stimulator was switched on to measure their baseline inhibitory response. The administration of WIN 

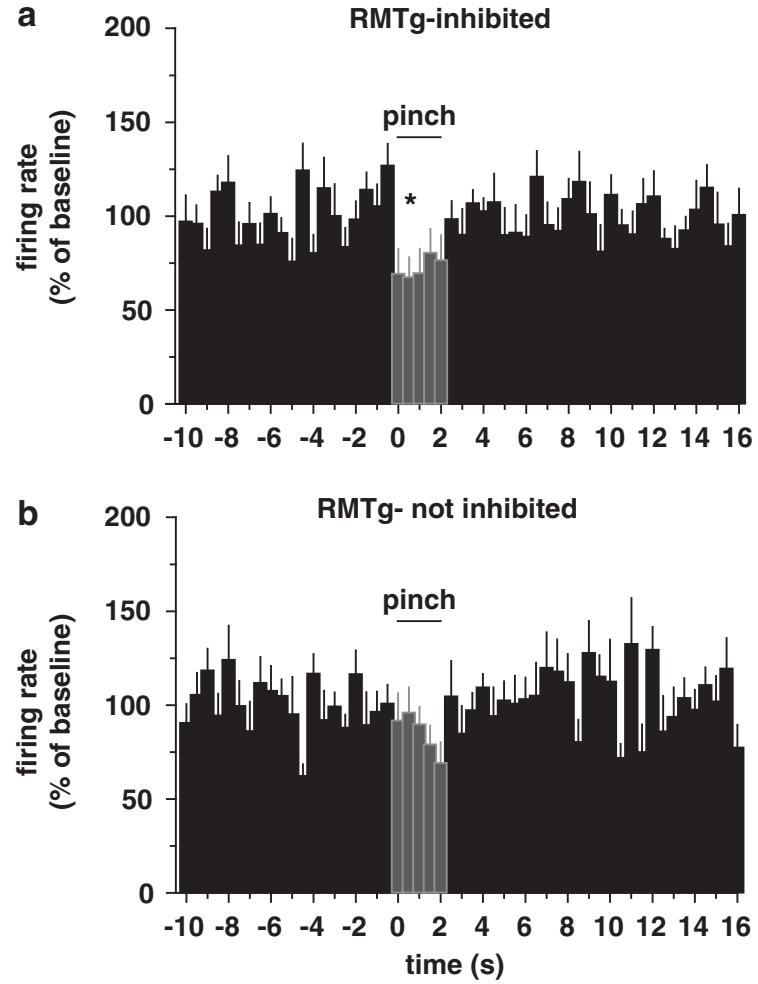

Figure 3 DA neurons inhibited by RMTg stimulation show a significant decrease in firing rate after paw pinch. (a) Average rate histogram (0.5 s bin) for 12 putative DA neurons suppressed from RMTg (RMTginhibited) illustrating the rapid and short-term decrease in firing frequency after paw pinch $(2 \mathrm{~s})$. (b) Conversely, DA cells whose activity was not suppressed by RMTg stimulation (RMTg-not inhibited) did not show any significant change in firing rate after the aversive stimulus $(n=8)$. ${ }^{*} P<0.05$, one-way ANOVA, and Dunnett's test.

$(0.5 \mathrm{mg} / \mathrm{kg}$, iv) was preceded $(200 \mathrm{~s})$ by the injection of either rimonabant $(0.5 \mathrm{mg} / \mathrm{kg}$, iv; $n=6)$ or its vehicle $(1 \mathrm{ml} / \mathrm{kg}$, iv; $n=7$; Figure $4 \mathrm{a}$ and b). Only one cell was recorded per rat, hence the number of animals used in this subset of experiments is 13. Two-way ANOVA for repeated measures of the time courses yielded a very significant treatment $\times$ time interaction $\left(F_{1,55}=10.67, P<0.0001\right)$, therefore separate analyses of drugs' effects were carried out. The administration of either vehicle or rimonabant did not affect the duration of RMTg-evoked inhibition of DA neurons (vehicle: $n=7, \mathrm{~F}_{2,12}=1.45, P=0.27$; rimonabant: $n=6, \mathrm{~F}_{2,10}=0.48, P=0.63$, one-way ANOVA for repeated measures; Figure 4b). On the other hand, WIN, when injected after vehicle, robustly reduced RMTg-induced suppression of VTA DA firing activity to $\sim 22 \%$ of baseline (baseline duration of inhibition: $94.0 \pm 10.4 \mathrm{~ms}$, maximum effect of WIN: $20.6 \pm 7.4 \mathrm{~ms} ; n=7, \mathrm{~F}_{3,18}=13.93, P<0.0001$, one-way ANOVA for repeated measures and Dunnett's test; Figure $4 \mathrm{a}$ and $\mathrm{b}$ ). This effect occurred quite rapidly (within the first $100 \mathrm{~s}$ from injection), persisted relatively unchanged over $20 \mathrm{~min}$, and required CB1 receptor activation. Indeed, rimonabant completely prevented WIN's effects when administered $200 \mathrm{~s}$ before the agonist (baseline duration of inhibition: $84.3 \pm 26.2 \mathrm{~ms}$, maximum effect of WIN: $81.1 \pm 26.2 \mathrm{~ms} ; n=6, \mathrm{~F}_{3,15}=0.06, P=0.98$, one-way ANOVA for repeated measures; Figure 4b). Additionally, in all experiments $(n=5)$ when the cell was recorded for a time sufficient to allow the administration of rimonabant 5 min after the agonist, rimonabant fully restored RMTginduced inhibition of VTA neuron activity to baseline levels (Figure $4 \mathrm{a}$ and $\mathrm{c}$ ). For these experiments, the comparison between baseline inhibition, the effect of WIN, and the effect of rimonabant (for both drugs' effects the time point analyzed was $300 \mathrm{~s}$ after injection) yielded a highly significant difference $\left(n=5, \mathrm{~F}_{2,8}=18.08, P=0.0011\right.$, oneway ANOVA for repeated measures, and Tukey's test; Figure $4 \mathrm{a}$ and c). Post hoc analysis revealed that postrimonabant inhibition was not different from baseline values $(P>0.05$, Tukey's test), but significantly different from post-WIN inhibition ( $P<0.01$, Tukey's test; Figure $4 \mathrm{c})$. These experiments confirm the involvement of CB1 receptors in WIN-induced suppression of inhibition and that the endocannabinoid system is not tonically activated.

$\mathrm{GABA}_{\mathrm{A}}$-mediated IPSCs recorded from VTA DA cells and evoked by repetitively stimulating caudal afferents (duration $50 \mu \mathrm{s}$, frequency $0.1 \mathrm{~Hz}$, intensity $0.01-0.3 \mathrm{~mA}$ ) in an in vitro midbrain slice preparation were supportive. Although we cannot identify definitively the sources of inhibitory afferents, we can assume that most of the caudal inputs electrically stimulated in our preparation are presumably originating from the RMTg, given that it is one of the main caudally located inhibitory sources to the VTA. The change in synaptic strength elicited by paired stimuli given at an interval of $50 \mathrm{~ms}$ was not different from those described before (Melis et al, 2002). In fact, DA cells exhibited a paired-pulse facilitation (IPSC2/IPSC1: $1.11 \pm$ $0.07, n=33$ ), which did not depend on the amplitude of the first IPSCs recorded (Figure $4 \mathrm{~d}$ ).

As CB1 receptor activation decreased RMTg neuron activity (Lecca et al, 2011) and RMTg-induced inhibition of DA cells, we examined whether WIN would affect caudal inhibitory transmission onto these neurons. WIN $(1 \mu \mathrm{M}, 5 \mathrm{~min})$ significantly and irreversibly reduced IPSCs amplitude by $57 \pm 5.2 \% \quad\left(n=5, \quad \mathrm{~F}_{19,80}=13.42\right.$, $P<0.0001$, one-way ANOVA for repeated measures; Figure 4d and e). Probably owing to its high lipophilicity, WIN effect did not wash out; however, when it was co-applied with two structurally different CB1 receptor antagonists, AM281 $(0.5 \mu \mathrm{M})$ and rimonabant $(1.0 \mu \mathrm{M})$, WIN-induced inhibition of IPSC amplitude was fully prevented (AM281: $n=5, \quad \mathrm{~F}_{1,152}=602.44, \quad P<0.0001$; rimonabant: $n=5$, $\mathrm{F}_{1,152}=23.47, \quad P<0.001$, two-way ANOVA for repeated measures; Figure 4e). As probability of release is inversely related to paired-pulse ratio, if WIN decreases IPSC amplitude through activation of presynaptic CB1 receptors, one would expect an increased paired-pulse ratio in the presence of this drug. WIN-induced decreased IPSC amplitude was accompanied by an increased pairedpulse ratio (from IPSC2/IPSC1 $=1.24 \pm 0.07$ to IPSC2/ IPSC1 $=1.53 \pm 0.03 ; n=5, P=0.01$, paired $t$-test; Figure $4 \mathrm{f}$ ). Furthermore, as analysis of the changes in quantitative measure of the variability (ie, $\mathrm{CV}^{2}$ ) of postsynaptic responses is a powerful, though indirect, tool to identify the locus for the modifications (Faber and Korn, 1991; Malinow and Tsien, 1990), we measured the changes in $\mathrm{CV}^{2}$ and evoked IPSCs caused by WIN (Figure 4f). Taken altogether, these data suggested that WIN reduced probability of GABA release presumably from RMTg terminals via activation of presynaptic CB1 receptors. 


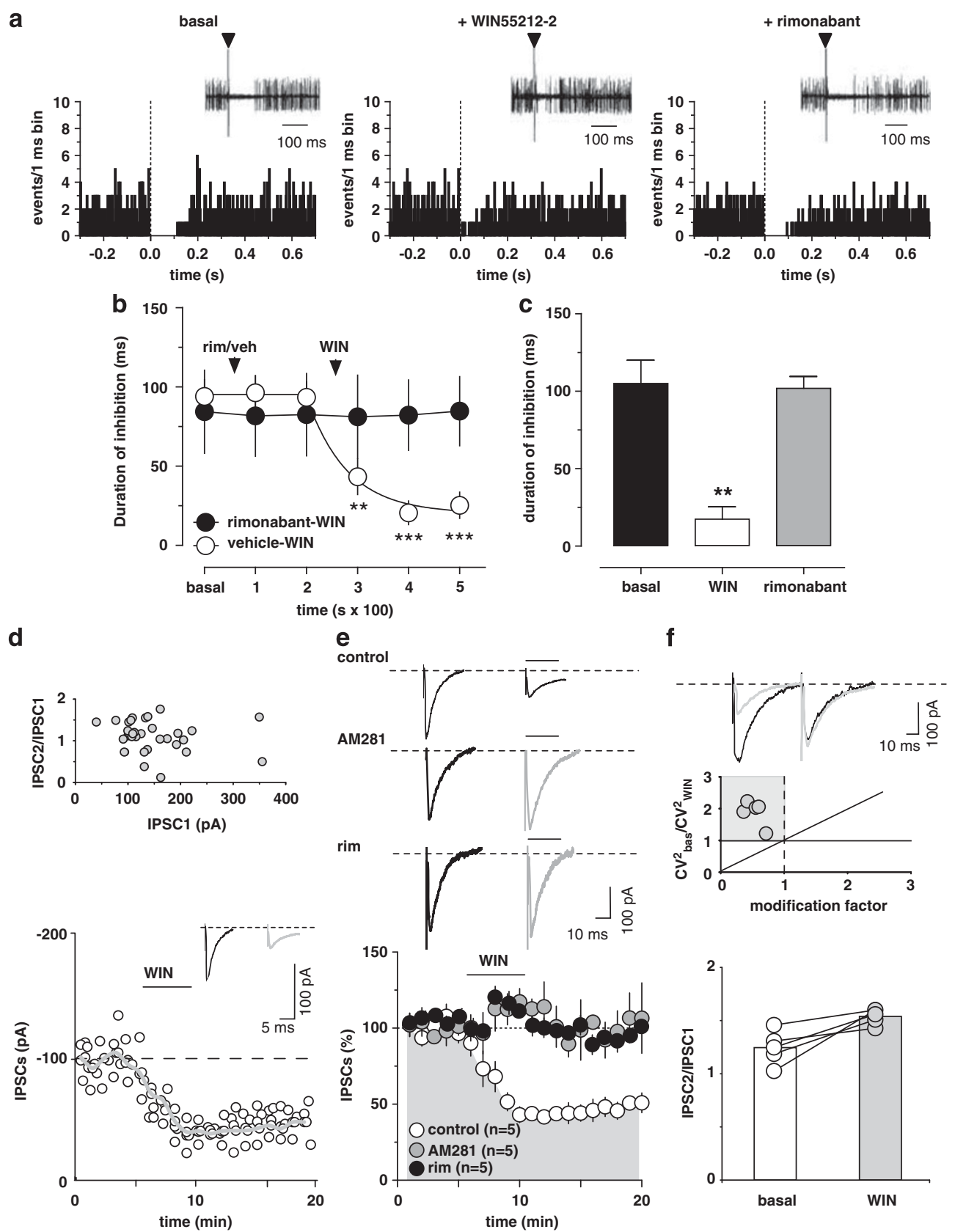

Figure 4 The cannabinoid receptor agonist WIN552I2-2 (WIN) blocks RMTg-evoked suppression of VTA DA neuron firing. (a) Representative PSTHs and superimposed traces acquired from a digital oscilloscope (insets) illustrating the typical inhibitory responses of VTA DA neurons after electrical stimulation of the RMTg $(\mathrm{I} \mathrm{Hz})$ at time 0 (arrowheads), the suppression of inhibition induced by WIN $(0.5 \mathrm{mg} / \mathrm{kg}$, iv), and the reversal by the CBI receptor antagonist rimonabant $(0.5 \mathrm{mg} / \mathrm{kg}$, iv). (b) This graph shows the time course of WIN's effect on RMTg-induced inhibition of VTA DA neurons, with ( $n=7$ ) and without $(n=6)$ rimonabant pretreatment. Rimonabant, injected before WIN, prevented the suppression of inhibition induced by the cannabinoid. (c) The bar graph summarizes the effects of WIN on the duration of inhibition evoked by RMTg stimulation and shows that rimonabant, administered 5 min after WIN, reverts the suppression of inhibition $(n=5)$. (d) WIN inhibits GABA synaptic transmission elicited by stimulation of caudal afferents in rat VTA DA cells. Top panel, no correlation was found between the amplitude of IPSCI and the IPSC2/IPSCI ratio. Each circle represents the mean paired-pulse ratio of 2 min recording. Bottom panel, a typical whole-cell voltage clamp recording showing that bath application of WIN (I $\mu$ M) inhibits IPSC amplitude, when cells are held at $-70 \mathrm{mV}$. The gray line represents mean IPSC amplitude. The inset shows single IPSC from a typical experiment, before (black line), and during (gray line) perfusion of WIN. (e) WIN reduces IPSC amplitude through activation of CBI receptors. All data are normalized to the respective baseline (5 min of baseline). Black bar shows time of superfusion of WIN, alone (open circles) and in the presence of CBI receptor antagonists AM28I (500 nM, gray circles), or rimonabant (I $\mu \mathrm{M}$, black circles). SEM bars are smaller than symbols in some cases. The top panel shows I2-trace averages of IPSCs in the absence (black line) and presence (gray line) of AM28I or rimonabant. Black bars represent time of WIN application. (f) Top panel, from the data presented in e, the ratio of CV in WIN to baseline is plotted against the modification factor (ie, the ratio of mean synaptic responses in WIN to baseline values). Data points in the gray area and along the diagonal line of $y=x$ imply a presynaptic reduction of synaptic transmission induced by WIN. Bottom panel, WIN enhances the paired-pulse ratio of IPSCs, producing paired-pulse facilitation. The circles show the paired-pulse ratio for each of the experiments in e before (open circles) and during (gray circles) the application of WIN, whereas the graph plots the averaged paired-pulse ratio in a bar graph form. Representative traces are shown in the inset, where the IPSCs are represented before (black lines) and during WIN (gray lines) application. The results are means, with vertical bars representing the SEM of duration of inhibition expressed as a percentage of the baseline. Arrows represent the time of injections. $* * P<0.0$ I, ****P<0.00 I, and one-way ANOVA. 
Morphine decreases RMTg-induced inhibition of VTA DA neurons. The most widely accepted hypothesis postulates that opiate excitation of VTA DA neurons results from disinhibition consequent to a primary inhibitory effect on local GABA interneurons (Johnson and North, 1992). Accordingly, in vivo morphine reduces GABA neuron discharge activity in both the VTA (Steffensen et al, 2006) and RMTg (Jalabert et al, 2011; Lecca et al, 2011). As RMTg afferents exert a tonic inhibitory influence on midbrain DA cells (Ikemoto, 2010; Lecca et al, 2011), we decided to assess whether morphine would also affect RMTg-evoked suppression of VTA DA spontaneous neuronal firing. We recorded DA cells in the VTA that showed an inhibitory response following RMTg stimulation $(0.5 \mathrm{~mA}, 0.5 \mathrm{~ms}, 1 \mathrm{~Hz}$, Figure 5a).

The administration of morphine $(4.0 \mathrm{mg} / \mathrm{kg}$, iv) was preceded $(200 s)$ by the injection of either naloxone $(0.1 \mathrm{mg} / \mathrm{kg}$, iv; $n=6)$ or its vehicle $(1 \mathrm{ml} / \mathrm{kg}, \mathrm{iv} ; n=7)$ (Figure $5 \mathrm{a}$ and $\mathrm{b}$ ). As only one cell was recorded per rat, the number of animal used in this subset of experiments is 13 . Two-way ANOVA analysis for repeated measures of the time courses yielded a very significant drug $\times$ time interaction $\left(F_{1,55}=3.46, P<0.0087\right)$, therefore separate analyses of drugs' effects were carried out. The administration of either vehicle or naloxone did not affect the duration of RMTg-evoked inhibition of DA neurons (vehicle: $n=7$, $\mathrm{F}_{2,12}=1.15, P=0.35$; naloxone: $n=6, \mathrm{~F}_{2,10}=3.049, P=0.09$, one-way ANOVA for repeated measures; Figure $5 b$ ). Conversely, morphine, when injected after vehicle, significantly shortened RMTg-induced suppression of VTA DA firing activity to $\sim 48 \%$ of baseline (baseline duration of inhibition: $81.7 \pm 18.2 \mathrm{~ms}$; maximum effect of morphine: $39.4 \pm 12.4 \mathrm{~ms} ; \quad n=7, \quad \mathrm{~F}_{3,18}=8.98, \quad P<0.001$, one-way ANOVA for repeated measures and Dunnett's test; Figure $5 \mathrm{a}$ and $\mathrm{b})$. This effect required $\mu$-opioid receptor activation, as naloxone prevented morphine's effects when administered $200 \mathrm{~s}$ before the agonist (baseline duration of inhibition: $92.3 \pm 20.5 \mathrm{~ms}$; effect of morphine at $300 \mathrm{~s}$ : $121.2 \pm 25.2 \mathrm{~ms} ; n=6, \mathrm{~F}_{3,15}=1.88, P=0.17$, one-way ANOVA for repeated measures; Figure $5 \mathrm{~b}$ ) and fully restored RMTginduced inhibition of VTA neuron activity to baseline levels in all experiments $(n=5)$ when it was injected after $(5 \mathrm{~min})$ the agonist (Figure 5a and c). For these experiments, the comparison between baseline inhibition, the effect of morphine, and the effect of naloxone (for both drugs' effects the time point analyzed was $300 \mathrm{~s}$ after injection) yielded a significant difference $\left(n=5, \mathrm{~F}_{2,8}=4.47, P<0.05\right.$, one-way ANOVA for repeated measures and Tukey's test; Figure 5a and c). These results indicate that the effects of morphine require the activation of opioid receptors and that endogenous opioids do not exert a significant tonic control over the RMTg-DA synapse.

Additionally, in order to investigate whether or not $\mu$-opioid receptor activation might affect inhibitory synapses arising from RMTg onto VTA DA cells, we measured $\mathrm{GABA}_{\mathrm{A}}$ IPSCs recorded from DA neurons. Acute bath application of morphine at a concentration of $1 \mu \mathrm{M}$ (3 min) significantly reduced IPSCs by $42 \pm 4.2 \%\left(n=5, \mathrm{~F}_{19,99}=\right.$ 13.59; $P<0.0001$; Figure $5 \mathrm{~d}$ and e), and the effect did not depend on the amplitude of the first IPSCs $(r=0.48$, $P>0.05$, data not shown). The effect of morphine was reversible on wash out, and it was fully abolished in the presence of naloxone $\left(0.1 \mu \mathrm{M} ; n=5, \mathrm{~F}_{1,152}=3.76, P=0.008\right.$, two-way ANOVA for repeated measures; Figure 5e). Because modifications in the paired-pulse ratio are considered to reflect changes in transmitter release (Melis et al, 2002; Nie et al, 2004), we analyzed whether morphine affected it. Acute bath application of morphine increased the pairedpulse ratio (from IPSC2/IPSC1 $=1.10 \pm 0.02$ to IPSC2/ IPSC1 $=1.63 \pm 0.11 ; \quad n=5, \quad P=0.005, \quad$ paired $t$-test; Figure 5f). Further analysis of the $\mathrm{CV}^{2}$ and evoked IPSCs caused by morphine (Figure $5 \mathrm{f}$ ) are indicative of a presynaptic locus for these modifications, consistently with previous reports (Lecca et al, 2011) and the present in vivo data.

Cocaine and nicotine do not affect the response of VTA $D A$ neurons to RMTg stimulation. We have previously shown that cocaine, similarly to WIN and morphine but to a lesser magnitude, inhibits RMTg discharge activity (Lecca et al, 2011). On the other hand, nicotine produces a strong and enduring stimulation on RMTg neuron firing rate (Lecca et al, 2011). Here, we sought to verify whether nicotine and cocaine interact with RMTg GABA inputs to the VTA. To test this hypothesis, we first assessed the effect of nicotine on seven DA neurons in the VTA (from seven rats) exhibiting an inhibitory response to RMTg stimulation (Figure 6a). As illustrated in Figure $6 \mathrm{a}$, nicotine $(0.2 \mathrm{mg} / \mathrm{kg}$, iv) administered $200 \mathrm{~s}$ after vehicle injection caused no change in the duration of RMTg-induced inhibition of VTA DA neuronal firing (baseline duration of inhibition: $72.1 \pm 15.1 \mathrm{~ms}$; effect of nicotine at $300 \mathrm{~s}: 75.3 \pm 13.7 ; n=7$, $\mathrm{F}_{5,30}=0.63, P=0.68$, one-way ANOVA for repeated measure; Figure 6a). Next, we examined cocaine's action on VTA inhibition from the RMTg. We selected a subset of seven DA neurons (from seven rats) whose spontaneous activity was suppressed by RMTg stimulation (Figure $6 \mathrm{~b}$ ). Acute cocaine injection also $(1 \mathrm{mg} / \mathrm{kg}$, iv) did not affect the inhibition of VTA DA discharge rate evoked by RMTg stimulation (baseline duration of inhibition: $72.1 \pm 15.1 \mathrm{~ms}$; effect of cocaine at $300 \mathrm{~s}: 75.8 \pm 16.5 ; n=7, \mathrm{~F}_{5,30}=0.22$, $P=0.94$, one-way ANOVA for repeated measure; Figure $6 \mathrm{~b}$ ). An example of the lack of effect of cocaine on RMTginduced VTA responses is shown in Figure $6 \mathrm{~b}$. As these drugs did not affect RMTg-induced inhibition in vivo, we did not further analyze their effects on evoked IPSCs with patch clamp experiments.

\section{DISCUSSION}

Our results provide the electrophysiological evidence of the monosynaptic inhibitory RMTg projections on VTA DA neurons, and the relationship between spontaneous discharge activity of these cells and the strength of RMTg inhibition. Moreover, we discovered that this inhibition could contribute to the effects of some drugs of abuse. Specifically, we showed that both cannabinoids and opioids share the property of strongly reducing the suppression of VTA neuronal activity elicited by the electrical stimulation of the RMTg in vivo, as well as to depress IPSCs evoked by caudal afferents in vitro. We therefore provide a plausible and alternative mechanism of action by which cannabinoids and opioids excite DA neurons, ie, inhibition of extrinsic GABA afferents arising from the RMTg. 



d

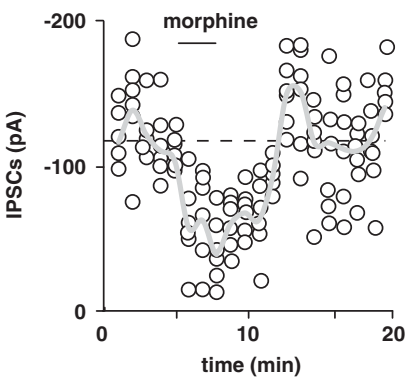

e control<smiles>COC(=O)C1CC1C1CC1C</smiles>

naloxone

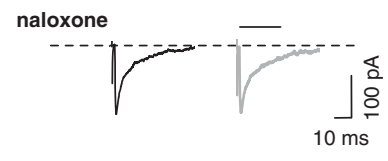



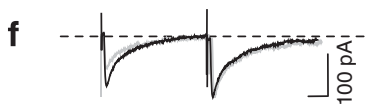

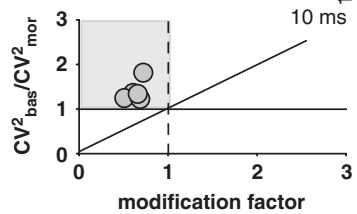

modification factor

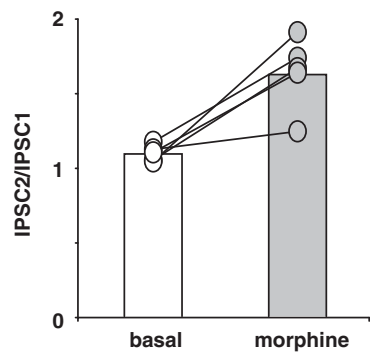

Figure 5 Morphine shortens the duration of RMTg-evoked inhibition of VTA DA neurons through activation of $\mu$-opioid receptors. (a) Representative PSTHs and superimposed traces acquired from a digital oscilloscope (insets) showing inhibitory responses elicited in DA neurons by RMTg stimulation ( $\mathrm{Hz}$, delivered at time 0 , arrowheads). Morphine $(4 \mathrm{mg} / \mathrm{kg}$, iv) reduced RMTg-evoked inhibition. The effect was fully reverted by the $\mu$-opioid antagonist naloxone $(0.1 \mathrm{mg} / \mathrm{kg}$, iv). (b) Time course of morphine's effects on RMTg-evoked suppression of VTA DA discharge activity, with and without ( $n=7$ ) naloxone pretreatment. Naloxone $(n \mid x)$ completely prevented morphine's reduction of inhibition $(n=6)$. (c) The bar graph summarizes the actions of morphine on the duration of VTA inhibition induced by RMTg stimulation and shows that naloxone $(n=5,5$ min after morphine) reverts morphine's effects. (d) Morphine induces inhibition of GABA synaptic transmission elicited by stimulation of RMTg afferents in rat VTA DA cells. A typical whole-cell patch clamp recording showing that bath application of morphine $(I \mu M)$ inhibits IPSC amplitude, when cells are held at $-70 \mathrm{mV}$. The gray line represents mean IPSC amplitude. (e) Morphine reduces IPSC amplitude through activation of $\mu$-opioid receptors. All data are normalized to the respective baseline (5 min of baseline). The black bar shows time of superfusion of morphine in the presence (gray circles) and absence (open circles) of the $\mu$-opioid antagonist naloxone ( 100 nM). SEM bars are smaller than symbols in some cases. The inset shows I2-trace averages of IPSCs in the absence (black line) and presence (gray line) of naloxone. Black bars represent time of morphine application. (f) Top panel, from the data presented in e, the ratio of $\mathrm{CV}^{2}$ in morphine to baseline is plotted against the modification factor (ie, the ratio of mean synaptic responses in morphine to baseline values). Data points in the gray area and along the diagonal line of $y=x$ imply a presynaptic reduction of synaptic transmission induced by morphine. Bottom panel, morphine enhances the paired-pulse ratio of IPSCs, producing paired-pulse facilitation. The circles show the paired-pulse ratio for each of the experiments in $f$ before (open circles) and during (gray circles) the application of morphine, whereas the graph plots the averaged paired-pulse ratio in a bar graph form. Representative traces are shown in the inset, where the IPSCs are represented before (black lines) and during morphine (grey lines) application. The results are means with vertical bars representing the SEM of the duration of inhibition expressed as a percentage of the baseline. Arrows represent the time of injections. $* P<0.05$, ** $P<0.001$, **** $P<0.0001$ vs baseline, and one-way ANOVA. 


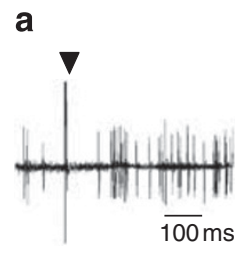

basal
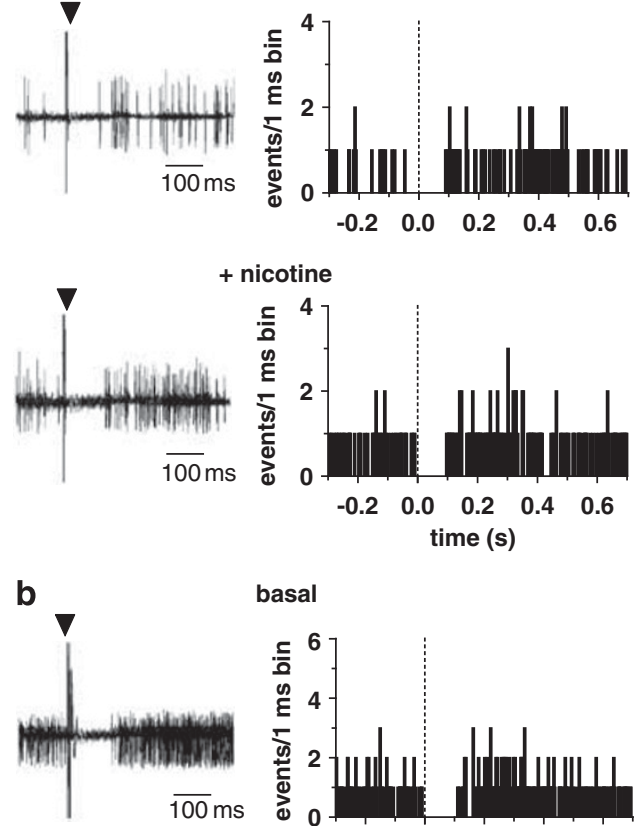

basal
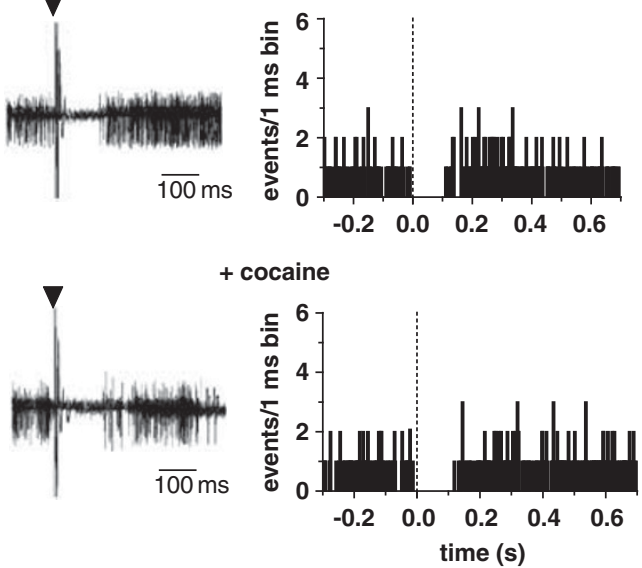


Figure 6 Nicotine and cocaine do not affect the suppression of VTA DA spontaneous activity elicited by RMTg stimulation. (a) Example of short-latency RMTg-evoked responses in a VTA DA neuron before and after nicotine $(0.2 \mathrm{mg} / \mathrm{kg}$, iv). Inhibitory responses were unchanged. On the right, the graph shows the time course of nicotine's effect on the duration of inhibition $(n=6)$. (b) Example of RMTg-evoked responses before and after cocaine in a VTA DA neuron. VTA inhibitory responses were also unaffected by cocaine $(\mathrm{I} \mathrm{mg} / \mathrm{kg}$, iv). The time course of cocaine's effect is shown (right panel). The results are means, with vertical bars representing the SEM of the duration of inhibition expressed as a percentage of the baseline $(n=5)$.

The physiological relevance of the RMTg as a primary source of inhibitory inputs to DA neurons is emphasized by the finding that RMTg stimulation induced a time-locked temporary cessation of discharge activity in half of DA cells of the VTA, thus suggesting a monosynaptic pathway. This result is in agreement with anatomical studies showing that about $60 \%$ of VTA DA neurons are directly innervated by RMTg GABA projections (Jhou et al, 2009a; Jhou et al, 2009b; Kaufling et al, 2010a). It must be pointed out, however, that this proportion could be underestimated, as the RMTg shifts dorsally as it extends caudalward, hence we unlikely stimulated the entire efferent projections. Hence, studies in monkeys have determined that $96 \%$ of DA neurons were inhibited by RMTg stimulation (Hong et al, 2011). Interestingly, the electrical stimulation of the LHb, one of the main excitatory inputs to RMTg, induces a similar inhibition, though relatively delayed, on a large number of VTA DA neurons (Christoph et al, 1986; Ji and Shepard, 2007). This is consistent with recent anatomical studies illustrating that glutamatergic projections from $\mathrm{LHb}$ primarily end on GABA neurons in the RMTg (BalcitaPedicino et al, 2011) and that inhibition of DA neurons is disynaptic. Furthermore, our characterization of the
RMTg-induced inhibition on DA neurons underscored a negative correlation between the spontaneous activity of DA neurons and the duration of activity suppression: the higher the firing rate, the shorter the time of complete firing cessation induced by RMTg stimulation. This result further suggests that RMTg afferents to VTA DA neurons are major regulators of their spontaneous discharge rate. Moreover, bursting and regularly firing cells were silenced by RMTg stimulation for a shorter duration than irregular cells, suggesting that the latter subpopulation of DA neurons is under a stronger control by the RMTg. Additionally, the reaction of DA neurons to a noxious stimulus (paw pinch) was crucially dependent on their response to the RMTg. Hence, only DA cells inhibited by RMTg stimulation were also significantly inhibited by paw pinch. This finding can be explained by the prominent role of RMTg in the encoding of aversive stimuli (Jhou et al, 2009a), by responding to paw pinch with a fast and phasic excitation (Lecca et al, 2011), whereas DA neurons display heterogeneous responses (Brischoux et al, 2009). Our results suggest the possibility that this heterogeneity might depend on the presence of RMTg synapses impinging on DA neurons and/ or their relative strength, and that DA neurons might also 
encode aversive and noxious signals depending on whether they are innervated by the RMTg. It must be pointed out, however, that conventional criteria for DA neurons identification are quite conservative (see Materials and Methods) and might have led us to exclude subpopulations of DA neurons that differ from the main population in several parameters, such as high firing rate or short duration of action potentials (Lammel et al, 2008; Margolis et al, 2008).

Our findings that synapses between RMTg neurons and DA cells could also be an important locus of action for some drugs of abuse support the notion that firing rate and pattern of VTA DA cells depend crucially on the balance between excitatory and inhibitory afferents (Marinelli et al, 2006; Morikawa and Paladini, 2011), rather than on their intrinsic properties, and that affects DA release in terminal regions.

Hence, our observation that WIN suppressed the inhibition of DA neurons evoked by RMTg stimulation, an effect reversed by the cannabinoid antagonist rimonabant, is consistent with the depression of IPSCs evoked by stimulation of caudal afferents through a reduced probability of GABA release. This effect is mediated by CB1 receptors expressed by GABA terminals in the VTA (Marinelli et al, 2007; Matyas et al, 2007; Wenger et al, 2003), where they decrease GABA release (Marinelli et al, 2007; Szabo et al, 2002). Taken together, the combined effects of RMTg inhibition (Lecca et al, 2011) and depression of its GABA synaptic inputs to DA neurons (present results) might provide the first evidence, to our knowledge, for the mechanism by which cannabinoids excite DA cells in vivo (French, 1997; Gessa et al, 1998).

Similarly, morphine not only decreased the duration of RMTg-induced inhibition on DA neurons, but also reduced the amplitude of IPSCs evoked by the stimulation of caudal afferents to the VTA. Our findings point to a presynaptic mechanism, which would imply the presence of presynaptic $\mu$-opioid receptors on GABA terminals arising from the RMTg. This is consistent with the presence of $\mu$-opioid receptors on RMTg neurons (Jalabert et al, 2011), and with the effect of morphine on RMTg neuronal activity (Lecca et al, 2011), that was accompanied by a reduction of excitatory postsynaptic current amplitude recorded from those neurons. Opioids inhibit GABA synaptic transmission by reducing the probability of presynaptic neurotransmitter release in several brain regions (Capogna et al, 1993; Vaughan and Christie, 1997a; Vaughan et al, 1997b), and the VTA does not make an exception to this rule (Bergevin et al, 2002). Thus, similar to cannabinoids, opioids might alternatively disinhibit VTA DA neurons by means of a depression of discharge activity of RMTg cells coupled with a reduced probability of GABA release through presynaptic $\mu$-opioid receptors.

Conversely, neither nicotine nor cocaine affects the duration of RMTg-evoked suppression of DA neurons in vivo. This was not unexpected, given that the mechanism of action of these drugs on DA neurons is not a disinhibition (Luscher and Ungless, 2006).

Previous studies have already shown that nicotineinduced increase of GABA drive to DA neurons, as indexed by an enhanced spontaneous IPSC frequency, is eliminated by tetrodotoxin (TTX; Keath et al, 2007). The TTX sensitivity of nicotine's effects indicates that an increase in action potential activity of GABA neurons innervating the VTA might be responsible. Consistently, we previously demonstrated that nicotine strongly enhances firing rate of RMTg neurons (Lecca et al, 2011). However, nicotine did not enhance the duration of DA cell inhibition, as one would have expected if GABA release was facilitated. The reason is probably due to the contextual nicotine-induced increase in glutamate release (Mansvelder et al, 2002), which functionally counteracts the effects on GABA.

On the other hand, cocaine increases DA release (Di Chiara and Imperato, 1988) and inhibits firing rate of DA neurons (Einhorn et al, 1988; Luchicchi et al, 2010) through blockade of the DA transporter. Other studies showed that in VTA DA neurons cocaine also reduced IPSC frequency (Steffensen et al, 2008) and suggested that this drug acts on GABA neurons to reduce activity-dependent GABA release on DA cells by blocking voltage-gated $\mathrm{Ca}^{2+}$ channels. This effect may synergize with the inhibition of the DA transporter to further enhance DA release (Steffensen et al, 2008). Our results show that cocaine, although it reduces the discharge activity of both RMTg and DA neurons, does not affect the inhibition induced by RMTg stimulation. This finding leads us to hypothesize that cocaine does not target the RMTg-DA synapses.

The fact that cocaine and nicotine do not affect the duration of RMTg-induced firing suppression of DA neurons, but they do decrease or increase, respectively, firing rate of these cells, strongly suggests that the effect of drugs on frequency and on the evoked inhibition are not passively correlated, and therefore that one is not a consequence of the other.

In conclusion, our results suggest that cannabinoids and opioids profoundly influence both RMTg neuron activity and synaptic responses of DA neurons evoked by RMTg activation. It can be speculated that RMTg neurons, which lie in functional opposition to reward-encoding DA cells, might take place in the complex neural circuits mediating aversion and reward.

\section{ACKNOWLEDGEMENTS}

We thank Stefano Aramo and Barbara Tuveri for their skillful technical assistance, and the Regione Autonoma della Sardegna, Assessorato alla Programmazione for the support given to MP (grants for basic research, Legge Regionale 7/2007) and to SL, and AL through the program 'Bursaries for Young Researchers' (Legge Regionale 7/2007).

\section{DISCLOSURE}

The authors declare no conflict of interest.

\section{REFERENCES}

Balcita-Pedicino JJ, Omelchenko N, Bell R, Sesack SR (2011). The inhibitory influence of the lateral habenula on midbrain dopamine cells: ultrastructural evidence for indirect mediation via the rostromedial mesopontine tegmental nucleus. J Comp Neurol 519: 1143-1164.

Bergevin A, Girardot D, Bourque MJ, Trudeau LE (2002). Presynaptic mu-opioid receptors regulate a late step of the 
secretory process in rat ventral tegmental area GABAergic neurons. Neuropharmacology 42: 1065-1078.

Bonci A, Williams JT (1997). Increased probability of GABA release during withdrawal from morphine. J Neurosci 17: 796-803.

Brischoux F, Chakraborty S, Brierley DI, Ungless MA (2009). Phasic excitation of dopamine neurons in ventral VTA by noxious stimuli. Proc Natl Acad Sci USA 106: 4894-4899.

Capogna M, Gahwiler BH, Thompson SM (1993). Mechanism of mu-opioid receptor-mediated presynaptic inhibition in the rat hippocampus in vitro. J Physiol 470: 539-558.

Christoph GR, Leonzio RJ, Wilcox KS (1986). Stimulation of the lateral habenula inhibits dopamine-containing neurons in the substantia nigra and ventral tegmental area of the rat. J Neurosci 6: 613-619.

Di Chiara G, Imperato A (1988). Drugs abused by humans preferentially increase synaptic dopamine concentrations in the mesolimbic system of freely moving rats. Proc Natl Acad Sci USA 85: $5274-5278$.

Einhorn LC, Johansen PA, White FJ (1988). Electrophysiological effects of cocaine in the mesoaccumbens dopamine system: studies in the ventral tegmental area. J Neurosci 8: 100-112.

Faber DS, Korn H (1991). Applicability of the coefficient of variation method for analyzing synaptic plasticity. Biophys $J \mathbf{6 0}$ : 1288-1294.

Fabricius K, Helboe L, Fink-Jensen A, Wortwein G, Steiniger-Brach B, Sotty F (2010). Increased dopaminergic activity in socially isolated rats: an electrophysiological study. Neurosci Lett 482: $117-122$.

Ford CP (2006). Properties and opioid inhibition of mesolimbic dopamine neurons vary according to target location. J Neurosci 26: $2788-2797$.

French ED (1997). delta9-Tetrahydrocannabinol excites rat VTA dopamine neurons through activation of cannabinoid CB1 but not opioid receptors. Neurosci Lett 226: 159-162.

French ED, Dillon K, Wu X (1997). Cannabinoids excite dopamine neurons in the ventral tegmentum and substantia nigra. Neuroreport 8: 649-652.

Geisler S, Marinelli M, Degarmo B, Becker ML, Freiman AJ, Beales $\mathrm{M}$ et al (2008). Prominent activation of brainstem and pallidal afferents of the ventral tegmental area by cocaine. Neuropsychopharmacology 33: 2688-2700.

Gessa GL, Melis M, Muntoni AL, Diana M (1998). Cannabinoids activate mesolimbic dopamine neurons by an action on cannabinoid CB1 receptors. Eur J Pharmacol 341: 39-44.

Grace AA, Bunney BS (1983). Intracellular and extracellular electrophysiology of nigral dopaminergic neurons-1. Identification and characterization. Neuroscience 10: 301-315.

Grace AA, Bunney BS (1984). The control of firing pattern in nigral dopamine neurons: burst firing. J Neurosci 4: 2877-2890.

Hong S, Jhou TC, Smith M, Saleem KS, Hikosaka O (2011). Negative reward signals from the lateral habenula to dopamine neurons are mediated by rostromedial tegmental nucleus in primates. J Neurosci 31: 11457-11471.

Hyland BI, Reynolds JN, Hay J, Perk CG, Miller R (2002). Firing modes of midbrain dopamine cells in the freely moving rat. Neuroscience 114: 475-492.

Ikemoto S (2010). Brain reward circuitry beyond the mesolimbic dopamine system: a neurobiological theory. Neurosci Biobehav Rev 35: 129-150.

Jalabert M, Bourdy R, Courtin J, Veinante P, Manzoni OJ, Barrot $M$ et al (2011). Neuronal circuits underlying acute morphine action on dopamine neurons. Proc Natl Acad Sci USA 108: $16446-16450$.

Jhou TC, Fields HL, Baxter MG, Saper CB, Holland PC (2009a). The rostromedial tegmental nucleus (RMTg), a GABAergic afferent to midbrain dopamine neurons, encodes aversive stimuli and inhibits motor responses. Neuron 61: 786-800.
Jhou TC, Geisler S, Marinelli M, Degarmo BA, Zahm DS (2009b). The mesopontine rostromedial tegmental nucleus: a structure targeted by the lateral habenula that projects to the ventral tegmental area of Tsai and substantia nigra compacta. J Comp Neurol 513: 566-596.

Ji H, Shepard PD (2007). Lateral habenula stimulation inhibits rat midbrain dopamine neurons through a GABA(A) receptormediated mechanism. J Neurosci 27: 6923-6930.

Johnson SW, North RA (1992). Opioids excite dopamine neurons by hyperpolarization of local interneurons. J Neurosci 12: 483-488.

Jones GA, Norris SK, Henderson Z (1999). Conduction velocities and membrane properties of different classes of rat septohippocampal neurons recorded in vitro. J Physiol 517( $\mathrm{Pt} 3$ ): 867-877.

Kaufling J, Veinante P, Pawlowski SA, Freund-Mercier MJ, Barrot $M$ (2009). Afferents to the GABAergic tail of the ventral tegmental area in the rat. J Comp Neurol 513: 597-621.

Kaufling J, Veinante P, Pawlowski SA, Freund-Mercier MJ, Barrot M (2010a). gamma-Aminobutyric acid cells with cocaineinduced DeltaFosB in the ventral tegmental area innervate mesolimbic neurons. Biol Psychiatry 67: 88-92.

Kaufling J, Waltisperger E, Bourdy R, Valera A, Veinante P, Freund-Mercier MJ et al (2010b). Pharmacological recruitment of the GABAergic tail of the ventral tegmental area by acute drug exposure. Br J Pharmacol 161: 1677-1691.

Keath JR, Iacoviello MP, Barrett LE, Mansvelder HD, McGehee DS (2007). Differential modulation by nicotine of substantia nigra $v s$ ventral tegmental area dopamine neurons. J Neurophysiol 98: 3388-3396.

Kelland MD, Chiodo LA, Freeman AS (1990). Anesthetic influences on the basal activity and pharmacological responsiveness of nigrostriatal dopamine neurons. Synapse 6: 207-209.

Lammel S, Hetzel A, Häckel O, Jones I, Liss B, Roeper J (2008). Unique properties of mesoprefrontal neurons within a dual mesocorticolimbic dopamine system. Neuron 57: 760-773.

Lecca S, Melis M, Luchicchi A, Ennas MG, Castelli MP, Muntoni AL et al (2011). Effects of drugs of abuse on putative rostromedial tegmental neurons, inhibitory afferents to midbrain dopamine cells. Neuropsychopharmacology 36: 589-602.

Luchicchi A, Lecca S, Carta S, Pillolla G, Muntoni AL, Yasar S et al (2010). Effects of fatty acid amide hydrolase inhibition on neuronal responses to nicotine, cocaine and morphine in the nucleus accumbens shell and ventral tegmental area: involvement of PPAR-alpha nuclear receptors. Addict Biol 15: 277-288.

Luscher C, Ungless MA (2006). The mechanistic classification of addictive drugs. PLoS Med 3: e437.

Malinow R, Tsien RW (1990). Presynaptic enhancement shown by whole-cell recordings of long-term potentiation in hippocampal slices. Nature 346: 177-180.

Mansvelder HD, Keath JR, McGehee DS (2002). Synaptic mechanisms underlie nicotine-induced excitability of brain reward areas. Neuron 33: 905-919.

Margolis EB, Mitchell JM, Ishikawa J, Hjelmstad GO, Fields HL (2008). Midbrain dopamine neurons: projection target determines action potential duration and dopamine $\mathrm{d} 2$ receptor inhibition. J Neurosci 28: 8908-8913.

Marinelli M, Rudick CN, Hu XT, White FJ (2006). Excitability of dopamine neurons: modulation and physiological consequences. CNS Neurol Disord Drug Targets 5: 79-97.

Marinelli S, Di Marzo V, Florenzano F, Fezza F, Viscomi MT, van der Stelt $\mathrm{M}$ et al (2007). N-arachidonoyl-dopamine tunes synaptic transmission onto dopaminergic neurons by activating both cannabinoid and vanilloid receptors. Neuropsychopharmacology 32: 298-308.

Matsumoto M, Hikosaka O (2007). Lateral habenula as a source of negative reward signals in dopamine neurons. Nature 447: 1111-1115. 
Matsumoto M, Hikosaka O (2009). Representation of negative motivational value in the primate lateral habenula. Nat Neurosci 12: $77-84$.

Matyas F, Urban GM, Watanabe M, Mackie K, Zimmer A, Freund TF et al (2007). Identification of the sites of 2-arachidonoylglycerol synthesis and action imply retrograde endocannabinoid signaling at both GABAergic and glutamatergic synapses in the ventral tegmental area. Neuropharmacology 54: 95-107.

Melis M, Camarini R, Ungless MA, Bonci A (2002). Long-lasting potentiation of GABAergic synapses in dopamine neurons after a single in vivo ethanol exposure. J Neurosci 22: 2074-2082.

Morikawa H, Paladini CA (2011). Dynamic regulation of midbrain dopamine neuron activity: intrinsic, synaptic, and plasticity mechanisms. Neuroscience 198: 95-111.

Nie Z, Schweitzer P, Roberts AJ, Madamba SG, Moore SD, Siggins GR (2004). Ethanol augments GABAergic transmission in the central amygdala via CRF1 receptors. Science 303: 1512-1514.

Paxinos G, Watson C (2007). The Rat Brain in Stereotaxic Coordinates, 7th edn. Elsevier Academic Press: London.

Perrotti LI, Bolanos CA, Choi KH, Russo SJ, Edwards S, Ulery PG et al (2005). DeltaFosB accumulates in a GABAergic cell population in the posterior tail of the ventral tegmental area after psychostimulant treatment. Eur J Neurosci 21: 2817-2824.

Pistis M, Perra S, Pillolla G, Melis M, Muntoni AL, Gessa GL (2004). Adolescent exposure to cannabinoids induces long- lasting changes in the response to drugs of abuse of rat midbrain dopamine neurons. Biol Psychiatry 56: 86-94.

Steffensen SC, Stobbs SH, Colago EE, Lee RS, Koob GF, Gallegos RA et al (2006). Contingent and non-contingent effects of heroin on mu-opioid receptor-containing ventral tegmental area GABA neurons. Exp Neurol 202: 139-151.

Steffensen SC, Taylor SR, Horton ML, Barber EN, Lyle LT, Stobbs SH et al (2008). Cocaine disinhibits dopamine neurons in the ventral tegmental area via use-dependent blockade of GABA neuron voltage-sensitive sodium channels. Eur J Neurosci 28: 2028-2040.

Szabo B, Siemes S, Wallmichrath I (2002). Inhibition of GABAergic neurotransmission in the ventral tegmental area by cannabinoids. Eur J Neurosci 15: 2057-2061.

Ungless MA, Magill PJ, Bolam JP (2004). Uniform inhibition of dopamine neurons in the ventral tegmental area by aversive stimuli. Science 303: 2040-2042.

Vaughan CW, Christie MJ (1997a). Presynaptic inhibitory action of opioids on synaptic transmission in the rat periaqueductal grey in vitro. $J$ Physiol 498(Pt 2): 463-472.

Vaughan CW, Ingram SL, Connor MA, Christie MJ (1997b). How opioids inhibit GABA-mediated neurotransmission. Nature 390: 611-614.

Wenger T, Moldrich G, Furst S (2003). Neuromorphological background of cannabis addiction. Brain Res Bull 61: 125-128.

Yamaguchi T, Wang HL, Li X, Ng TH, Morales M (2011). Mesocorticolimbic glutamatergic pathway. J Neurosci 31: 8476-8490. 